ARTICLE

\title{
A quantum heat engine driven by atomic collisions
}

\author{
Quentin Bouton ${ }^{1,3}$, Jens Nettersheim ${ }^{1,3}$, Sabrina Burgardt ${ }^{1}$, Daniel Adam¹, Eric Lutz ${ }^{2} \&$ Artur Widera (i) $^{1 凶}$
}

Quantum heat engines are subjected to quantum fluctuations related to their discrete energy spectra. Such fluctuations question the reliable operation of thermal machines in the quantum regime. Here, we realize an endoreversible quantum Otto cycle in the large quasi-spin states of Cesium impurities immersed in an ultracold Rubidium bath. Endoreversible machines are internally reversible and irreversible losses only occur via thermal contact. We employ quantum control to regulate the direction of heat transfer that occurs via inelastic spinexchange collisions. We further use full-counting statistics of individual atoms to monitor quantized heat exchange between engine and bath at the level of single quanta, and additionally evaluate average and variance of the power output. We optimize the performance as well as the stability of the quantum heat engine, achieving high efficiency, large power output and small power output fluctuations.

\footnotetext{
${ }^{1}$ Department of Physics and Research Center OPTIMAS, Technische Universität Kaiserslautern, Kaiserslautern, Germany. ${ }^{2}$ Institute for Theoretical Physics I, University of Stuttgart, Stuttgart, Germany. ${ }^{3}$ These authors contributed equally: Quentin Bouton, Jens Nettersheim. ${ }^{凶}$ email: widera@physik.uni-kl.de
} 
$\mathrm{M}$ ost engines used in modern society are heat engines. Such machines generate motion by converting thermal energy into mechanical work ${ }^{1}$. Two central figures of merit of heat engines are efficiency, defined as the ratio of workoutput and heat input, and power characterizing the work-output rate. Heat engines should ideally have high efficiency, large power output, and be stable, i.e., exhibit small power fluctuations. However, real thermal machines operate far from reversible conditions and their performance is thus reduced by irreversible $\operatorname{losses}^{2,3}$. At the same time, microscopic motors are exposed to thermal fluctuations and, at low enough temperatures, to additional quantum fluctuations, which are associated with random transitions between discrete energy levels. Both fluctuation mechanisms contribute to their instability ${ }^{4,5}$. An important issue is hence to design and optimize small heat engines in order to maximize both their performance and their stability ${ }^{6}$.

Nanoscopic heat engines have been implemented recently using a single trapped ion ${ }^{7}$ and a spin coupled to the single-ion motion $^{8,9}$. Indications for quantum effects have been reported in a spin engine consisting of nitrogen-vacancy centers interacting with a light field ${ }^{10}$, and quantum heat engine operation has been shown in nuclear magnetic resonance ${ }^{11,12}$ and single-ion ${ }^{9}$ systems. These thermal machines are based on harmonic oscillators or two-level systems, and the baths mediating heat exchange are simulated by interaction with either laser fields ${ }^{7-10}$ or radiofrequency pulses ${ }^{11,12}$.

We here experimentally realize a quantum Otto cycle using a large quasi-spin system in individual Cesium (Cs) atoms immersed in a quantum heat bath made of ultracold Rubidium $(\mathrm{Rb})$ atoms. Expansion and compression steps are implemented by varying an external magnetic field, changing the energy-level spacing of the engine and performing work ${ }^{13}$. Heat exchange between system and bath occurs via inelastic endoenergetic and exoenergetic spin-exchange collisions ${ }^{14}$. The increased number of internal engine states, compared to simple two-level systems, allows for high-energy turnover per cycle, while their finite number naturally limits power fluctuations due to saturation, in contrast to the unbounded spectrum of harmonic oscillators. We employ quantum control of the coherent spin-exchange process ${ }^{15}$ to control the direction of heat transfer between system and bath at the level of individual quanta of heat ${ }^{14}$, independently of the kinetic thermal state of the bath. The precise control of the spin states of both engine and bath effectively suppresses internal irreversible losses in individual collisions, and thus makes the quantum heat engine endoreversible. Endoreversible machines operate internally without dissipation, while (external) irreversible losses only occur via the contact with the bath ${ }^{2,3}$. They hence outperform fully irreversible engines and have played for this reason a central role in finite-time thermodynamics for forty years ${ }^{2,3}$. We note that quantum systems generally exhibit internal friction when their Hamiltonian does not commute at different times $^{16,17}$. We additionally characterize the discrete quantum heat transfer at the level of individual quanta using full-counting statistics $^{18,19}$ and monitor the population dynamics of the engine from single-atom and time-resolved measurements of the engine's quasi-spin distribution along the cycle. We employ this system and techniques to evaluate and optimize the performance as well as the stability of the quantum heat engine, achieving high efficiency, large power output and small power output fluctuations.

\section{Results}

Components of the neutral-atom machine. We experimentally immerse up to ten laser-cooled Cs atoms in the $\mid F_{\mathrm{Cs}}=3, m_{F, \mathrm{Cs}}=$ 3) state into an ultracold $\mathrm{Rb}$ gas of up to $10^{4}$ atoms in the state
$\left|F_{\mathrm{Rb}}=1, m_{F, \mathrm{Rb}}=-1\right\rangle$, both species confined in a common optical dipole trap (Fig. 1a) (Methods A). Here $F$ and $m_{F}$ denote the total atomic angular momentum and its projection onto the quantization axis, respectively. The quantization axis is given by an external magnetic field of $B_{1}=346.5 \pm 0.2 \mathrm{mG}$ or $B_{2}=31.6 \pm 0.1 \mathrm{mG}$. The Cs atoms quickly thermalize to the kinetic temperature of $T=$ $950 \pm 50 \mathrm{nK}$ of the gas. We operate the quantum heat engine in the spin-state manifold of the seven Cs hyperfine ground states $\left|F_{\mathrm{Cs}}=3, m_{F, \mathrm{Cs}}\right\rangle, m_{F, \mathrm{Cs}} \in[+3,+2, \ldots,-3]$, which define its quasispin. These states are energetically equally spaced with Zeeman energy $E_{n}^{\mathrm{Cs}}=n \lambda B$, with $\lambda=\left|g_{F}^{\mathrm{Cs}}\right| \mu_{\mathrm{B}}$, where $g_{F}^{\mathrm{Cs}}=-1 / 4$ is the Cs Landé factor, $\mu_{\mathrm{B}}$ Bohr's magneton and $n=3-m_{F, \mathrm{Cs}}{ }^{20}$, with the zero-point of energy set to the lowest-energy state $\left|m_{F, C s}=3\right\rangle$.

Heat between the quantum engine and the bath is exchanged at the microscopic level via inelastic spin-exchange collisions (Fig. 1c). Each collision changes the value of the quasi-spin of the $C$ s engine by $\Delta m_{\mathrm{Cs}}=\mp 1 \hbar$, leading to an energy change of $\Delta E^{\mathrm{Cs}}= \pm \lambda B$ for each Cs atom, and $\Delta m_{\mathrm{Rb}}= \pm 1 \hbar$ for one $\mathrm{Rb}$ atom corresponding to the energy change $\Delta E^{\mathrm{Rb}}=\mp \kappa B$, with $\kappa=\left|g_{F}^{\mathrm{Rb}}\right| \mu_{\mathrm{B}}$, where $g_{F}^{\mathrm{Rb}}=$ $-1 / 2$ is the $\mathrm{Rb}$ Landé factor ${ }^{14}$. The spin population thus directly reflects the energy exchange between engine and reservoir at the level of single energy quanta. The direction of the heat transfer is determined by the spin polarization of the $\mathrm{Rb}$ bath and by angular momentum conservation during individual collisions ${ }^{14}$. The spin polarization of the $\mathrm{Rb}$ atoms distinguishes a high-energy bath for $m_{\mathrm{Rb}}=-1$ from a low-energy bath for $m_{\mathrm{Rb}}=+1$. Control over the internal $\mathrm{Rb}$ state accordingly permits to either increase or decrease the energy of the quasi-spin of the engine. Heat exchange automatically stops after six spin-exchange collisions, because then the highest/lowest energy state has been reached. One collision transfers the colliding $\mathrm{Rb}$ atom to the $\left|F_{\mathrm{Rb}}=1, m_{F, \mathrm{Rb}}=0\right\rangle$ state, which forms the exhaust of the engine. Owing to the massive imbalance between the $\mathrm{Rb}$ and $\mathrm{Cs}$ atom numbers $\left(N_{\mathrm{Rb}} / N_{\mathrm{Cs}}>1000\right)$, the probability of a second collision with the same $\mathrm{Rb}$ atom is indeed vanishingly small. Memory effects are furthermore negligible, making the bath Markovian.

Implementation of the quantum Otto cycle. The quantum Otto cycle consists of four parts: one compression and one expansion step, during which work is performed, and a heating and a cooling stage, during which heat is exchanged ${ }^{13}$. The corresponding experimental sequence is shown in Fig. 1b. The Cs machine is first driven by up to six spin-exchange collisions into energetically higher states (at magnetic field $B_{1}$ ), absorbing average heat $\left\langle Q_{\mathrm{H}}\right\rangle$ in time $\tau_{\mathrm{H}}=t_{\mathrm{B}}$. Mean work $\left\langle W_{\mathrm{BC}}\right\rangle$ is then performed by adiabatically decreasing the magnetic field to $B_{2}$ in $\tau=t_{\mathrm{C}}-t_{\mathrm{B}}=10 \mathrm{~ms}$. This time is much longer than the inverse energy splitting $\Delta E$ of the quasi-spin states, making the process adiabatic. It is, however, fast enough to avoid unwanted spinexchange collisions, implying that no heat is transferred. The engine is subsequently brought into contact with the low-energy bath by flipping the spins of the Rb bath using microwave (MW) sweeps. The Cs engine is accordingly driven by up to six spinexchange collisions into energetically lower states, releasing heat $\left\langle Q_{\mathrm{C}}\right\rangle$ in time $\tau_{\mathrm{C}}=t_{\mathrm{D}}-t_{\mathrm{C}}$. Work $\left\langle W_{\mathrm{DA}}\right\rangle$ is further performed by adiabatically increasing the magnetic field back to $B_{1}$ in $\tau=t_{\mathrm{A}}-$ $t_{\mathrm{D}}=10 \mathrm{~ms}$. The $\mathrm{Rb}$ spins are finally flipped to their initial state with other microwave sweeps, restoring the high-energy bath.

While each single collision is coherent and thus amenable to quantum control ${ }^{15}$, coupling of the engine to the large number of bath modes in elastic collisions destroys the coherence between the engine's quasi-spin levels. Heat is thus associated with changes of occupation probabilities, $\langle Q\rangle=\sum_{n} E_{n} \Delta p_{n}$, whereas work corresponds to changes of energy levels, $\langle W\rangle=\sum_{n} p_{n} \Delta E_{n}{ }^{13}$. In our system, we concretely have $\left\langle Q_{\mathrm{H}}\right\rangle=\sum_{n} n\left(p_{n}^{\mathrm{B}}-p_{n}^{\mathrm{A}}\right) \lambda B_{1}$ for 

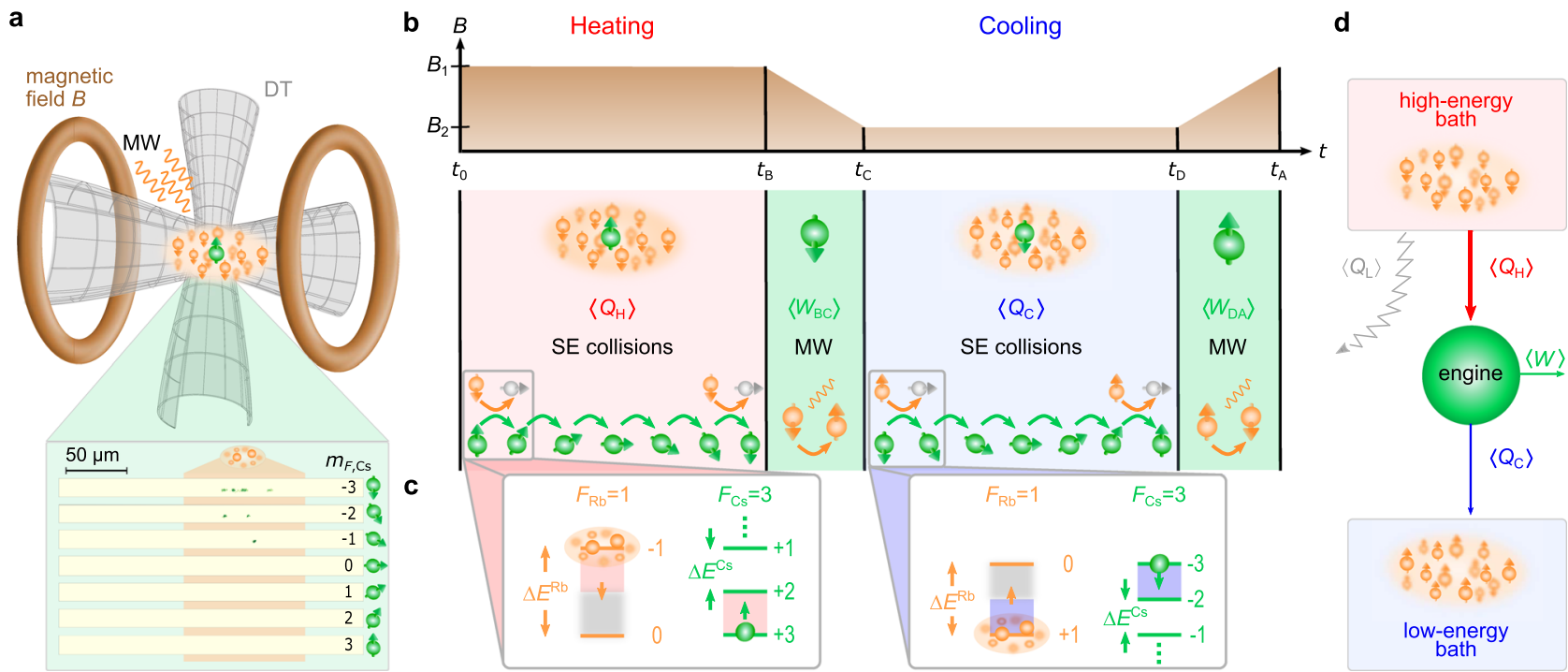

Fig. 1 Operation principle of the quantum heat engine. a Individual laser-cooled Cs atoms (green) are immersed in an ultracold Rb cloud (orange); both are confined in a common optical dipole trap (DT). External magnetic fields and microwave (MW) radiation, respectively, implement the power strokes of the quantum heat engine and distinguish the high- from the low-energy bath. The inset shows typical $m_{F}$-resolved fluorescence images of single $C s$ atoms for $t=t_{B}=300 \mathrm{~ms}$ after initialization, from which the quantized spin, and thus heat exchange, can be determined. The position of the bath cloud is indicated in orange with a width of $4 \sigma . \mathbf{b}$ The experimental Otto cycle consists of a heating stage, during which average heat $\left\langle Q_{H}\right\rangle$ is absorbed, and a power stroke induced by an adiabatic change of the magnetic field. A microwave field then switches the bath from high to low energy. The cycle is further completed by a cooling step, during which average heat $\left\langle Q_{c}\right\rangle$ is released, and an additional power stroke when the magnetic field is adiabatically brought back to its initial value. c The heat transfer between the Cs atom (engine) and a Rb (bath) atom occurs via inelastic spin-exchange collisions. In each collision, a single quantum of spin associated with a certain energy quantum is exchanged. Spin polarization of the Rb atoms and spin-conservation in individual collisions allow only up to six exo- or endothermal processes, corresponding to heating or cooling. $\mathbf{d}$ Owing to the difference of atomic Landé factors between $\mathrm{CS}$ and $\mathrm{Rb}$, the quantum heat engine (green) absorbs heat $\left\langle Q_{H}\right\rangle$ and releases heat $\left\langle Q_{C}\right\rangle$ (to produce work $\langle W\rangle$ ), while the bath releases more energy. The lost energy is irreversibly dissipated during an average of ten elastic collisions and is described by a heat leak $\left\langle Q_{L}\right\rangle$ from the highenergy bath.

heating and $\left\langle Q_{\mathrm{C}}\right\rangle=\sum_{n} n\left(p_{n}^{\mathrm{D}}-p_{n}^{\mathrm{C}}\right) \lambda B_{2}$ for cooling. On the other hand, the respective work contributions for expansion and compression are given by $\left\langle W_{\mathrm{BC}}\right\rangle=\sum_{n} n p_{n}^{\mathrm{B}} \lambda\left(B_{2}-B_{1}\right)$ and $\left\langle W_{\mathrm{DA}}\right\rangle=\sum_{n} n p_{n}^{\mathrm{D}} \lambda\left(B_{1}-B_{2}\right)$. In order to evaluate these average quantities, we determine the magnetic fields $B_{1}$ and $B_{2}$ with the help of Rb microwave spectroscopy (Methods). We further detect the Zeeman populations $p_{n}^{i}$ of single Cs atoms at arbitrary times by position resolved fluorescence measurements combined with Zeeman-state-selective operations (Fig. 1a inset) ${ }^{21}$. From each individual measurement, we can determine quantized spin transitions for each single atom. This allows us to monitor the resulting quantized heat exchange between engine and environment with a resolution of single quanta at each time. From a series of such measurements, we can further construct the average evolution of the quasi-spin populations (Fig. 2a, b): the progressive transfer from low (high) energy states to high (low) energy states during heating (cooling) as a function of time is clearly seen (green dots). From the measured heat counting statistics, we compute average (blue and red dots) and variance of heat exchange. We will use these quantities to examine the power output of the quantum machine and its fluctuations.

Performance of the quantum heat engine. We first characterize the performance of the quantum Otto engine by evaluating its efficiency given by ${ }^{13}$,

$$
\eta=\frac{\left\langle Q_{\mathrm{H}}\right\rangle-\left|\left\langle Q_{\mathrm{C}}\right\rangle\right|}{\left\langle Q_{\mathrm{H}}\right\rangle+\left\langle Q_{\mathrm{L}}\right\rangle}
$$

where $\left\langle Q_{\mathrm{H}}\right\rangle-\left|\left\langle Q_{\mathrm{C}}\right\rangle\right|$ is the total work produced by the machine, $\left\langle Q_{\mathrm{L}}\right\rangle$ the energy dissipated during the total heat exchange in one cycle, and $\left\langle Q_{\mathrm{H}}\right\rangle+\left\langle Q_{\mathrm{L}}\right\rangle$ the heat emitted by the high-energy bath
(Fig. 1d). Indeed, due to the different atomic Landé factors for Rb $\left(g_{F}^{\mathrm{Rb}}=-1 / 4\right) \quad$ and $\mathrm{Cs} \quad\left(g_{F}^{\mathrm{Cs}}=-1 / 2\right), \quad$ only half $\left(\gamma=g_{F}^{\mathrm{Cs}} / g_{F}^{\mathrm{Rb}}=1 / 2\right)$ of the energy change of a bath atom is effectively exchanged with the heat engine during an inelastic spin-exchange collision ${ }^{21}$. As a result, the heat emitted (absorbed) by the bath differs from the energy portions absorbed $\left\langle Q_{H}\right\rangle$ (emitted $\left\langle Q_{\mathrm{C}}\right\rangle$ ) by the machine. We macroscopically account for the remaining lost energy, which is irreversibly transferred to the kinetic energy of $\mathrm{Rb}$ during an average of ten elastic collisions, by a heat leak ${ }^{22}$ equal to $\left\langle Q_{\mathrm{L}}\right\rangle=\sum_{n} n\left(p_{n}^{B}-p_{n}^{A}\right) \kappa(1-\gamma)\left(B_{1}-B_{2}\right)$ with $\gamma=\lambda / \kappa$ the ratio of the Landé factors (Methods). We then obtain,

$$
\eta=\frac{\gamma\left(B_{1}-B_{2}\right)}{B_{1}-B_{2}+\gamma B_{2}} \leq 1-\frac{B_{2}}{B_{1}}=\eta_{\max }
$$

Its maximum value $\eta_{\max }$, reached in the absence of irreversible losses $(\gamma=1)$, is determined by the ratio of the two magnetic fields. We evaluate the efficiency (2) using experimental data for different cycle durations, $\tau_{\text {cycle }}=\tau_{\mathrm{H}}+\tau_{\mathrm{C}}+2 \tau$, by varying the heating and cooling times and evaluating the average heats $\left\langle Q_{\mathrm{H}}\right\rangle$ and $\left\langle Q_{C}\right\rangle$ (Fig. 3a). We find a constant value, i.e., independent of the number of spin-exchange collisions, of $\eta=0.478 \pm 0.002$. We emphasize that the internal efficiency of the quantum Otto engine, $\eta_{\text {int }}=1-\left|\left\langle Q_{\mathrm{C}}\right\rangle\right| /\left\langle Q_{\mathrm{H}}\right\rangle=0.917 \pm 0.009$ (Methods $\mathrm{C}$ ) is close to the maximal value $\eta_{\max }=0.908$. We may, therefore, conclude that irreversible losses mainly occur during heat transfer, while the engine itself runs reversibly. The quantum heat engine is hence endoreversible. We further note that, since heat losses are determined by the value of the Landé factors, they can in principle be reduced by choosing different atomic species. 


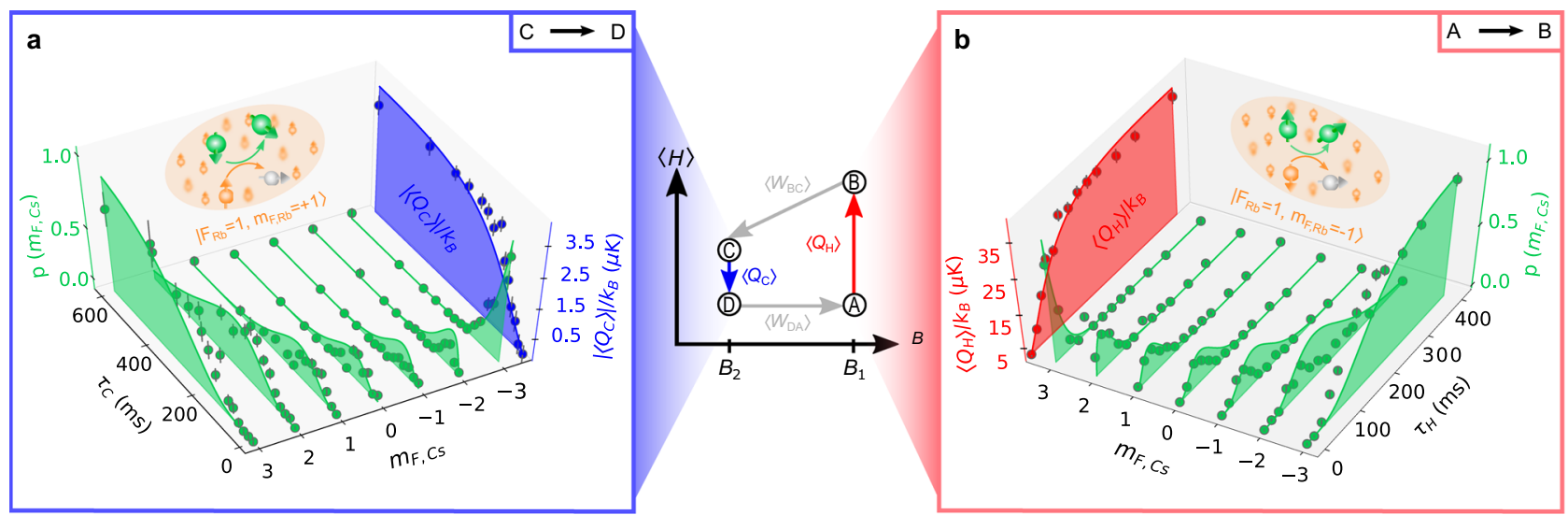

Fig. 2 Full-counting statistics of heat exchange. During the heating (AB) and cooling (CD) steps of the quantum Otto cycle (center), heat is exchanged with the bath. The average population dynamics of the individual engine levels are shown in green. The mean heats, $\left\langle Q_{C}\right\rangle$ and $\left\langle Q_{H}\right\rangle$, extracted from the fullcounting statistics are indicated for $\mathbf{a}$ cooling (blue) and $\mathbf{b}$ heating (red), as a function of the respective times $\tau_{\mathrm{C}}$ and $\tau_{\mathrm{H}}$. Dots show the experimental data, solid lines are a prediction of a microscopic model (Methods). In both panels, the population dynamics shows the transition from an initially spin-polarized engine state via a state of many populated $m_{F}$ levels to a spin-polarized state of the other extreme spin state. The inversion of an initially fully polarized population $\left(\left|m_{F, C s}=3\right\rangle \leftrightarrow\left|m_{F, C s}=-3\right\rangle\right)$ requires some hundreds of milliseconds. Error bars show statistical uncertainty of $\pm 1 \sigma$ standard deviation.

Second, we consider the average power of the quantum heat engine which reads,

$$
\langle P\rangle=\frac{\left\langle Q_{\mathrm{H}}\right\rangle-\left|\left\langle Q_{\mathrm{C}}\right\rangle\right|}{\tau_{\text {cycle }}} \leq \frac{\left\langle Q_{\mathrm{H}}\right\rangle}{\tau_{\text {cycle }}}\left(1-\frac{B_{2}}{B_{1}}\right) .
$$

We use the heat counting statistics to track its time evolution in Fig. 3b. We observe that the power (blue dots) increases with the number of inelastic collisions and reaches a maximum, $\langle P\rangle_{\max } / k_{\mathrm{B}}=30 \mathrm{nK} / \mathrm{ms}$, for a cycle time of $960 \mathrm{~ms}$. The corresponding number of inelastic collisions responsible for the heat exchange is almost 12 collisions total (6 spin-exchange collisions for the heating process and 6 for the cooling). This maximum nearly coincides with full population inversion between these two processes $\left(\left|m_{F, \mathrm{Cs}}=3\right\rangle \leftrightarrow\left|m_{F, \mathrm{Cs}}=-3\right\rangle\right)$, in analogy to that of a laser. Good agreement with a theoretical model (red solid line) is observed (Methods). From a collisional perspective, the energy transfer with the atomic bath is optimal in the sense that it exchanges the maximum energy of six quanta, which can be stored in the machine, in exactly six spin-exchange collisions as a consequence of the precise control of the spin states of machine and bath. The value of $\langle P\rangle_{\max }$ may be further optimized by enhancing the magnetic field difference, as well as the collision rate and the collision cross-section by controlling the temperature or density of the $\mathrm{Rb}$ gas.

We finally investigate the stability of the quantum Otto engine by analyzing the relative power fluctuations via the Fano factor, which quantifies the deviation from a Poisson distribution ${ }^{23}$,

$$
F_{P}=\frac{\sigma_{P}^{2}}{\langle P\rangle}=\frac{\left\langle P^{2}\right\rangle-\langle P\rangle^{2}}{\langle P\rangle},
$$

where $\sigma_{P}^{2}$ is the variance of the power, which we determine from the measured quasi-spin distributions (Methods). Figure $3 \mathrm{c}$ displays the Fano factor as a function of the cycle time, with the absolute fluctuations $\sigma_{P}$ shown in the inset. We find superPoissonian fluctuations $\left(F_{P}>1\right)$ for short cycle times, indicating that the quantum engine is unstable in this regime, with large relative power fluctuations. However, with increasing cycle time, the power increases faster than its variance, leading to a decrease in relative fluctuations. The transition to a Poissonian statistics $\left(F_{P}=1\right)$ (red dashed line), with strongly reduced power fluctuations and significantly increased stability, is located approximately at maximum power. This behavior follows from the finite Hilbert space of the Cs machine and the saturation effect due to the existence of an upper energy level. Importantly, the latter effect causes even the absolute value of the power fluctuations to decrease after on average six collisions (Fig. 3c inset). Power fluctuations could, in principle, also become sub-Poissonian $\left(F_{P}<\right.$ 1 ), but this regime is not seen experimentally due to experimental imperfections.

\section{Discussion}

In conclusion, we have realized an endoreversible quantum Otto cycle using single $\mathrm{Cs}$ atoms interacting with a $\mathrm{Rb}$ bath. The key asset of this machine is the exquisite control over both the fewlevel engine and the atomic reservoir. This unique feature allows us not only to regulate and monitor the heat exchange between system and environment at the single-quantum level, but also to operate the quantum engine in a regime of high efficiency, large power output and small power output fluctuations. The produced work could in principle be extracted by, e.g., coupling the magnetic moment of another microscopic particle to the magnetic moment of the engine. In a magnetic field gradient, motion of the coupled microscopic particle will directly reveal the work performed. Our system provides a versatile experimental platform to elucidate fundamental new effects generated by quantum reservoir engineering, such as nonequilibrium atomic baths ${ }^{24,25}$ and squeezed baths ${ }^{26,27}$, as well non-Markovian heat reservoirs by reducing the size of the $\mathrm{Rb}$ cloud ${ }^{28,29}$.

\section{Methods}

Experimental procedures. We start our experimental sequence by preparing an ultracold $\mathrm{Rb}$ gas in the magnetic field insensitive state $\left|F_{\mathrm{Rb}}=1, m_{F, \mathrm{Rb}}=0\right\rangle$ and, at a distance of $\approx 200 \mu \mathrm{m}$, a small sample of laser-cooled Cs atoms. The Cs atoms are further cooled and optically pumped into the $\left|F_{\mathrm{Cs}}=3, m_{F, \mathrm{Cs}}=3\right\rangle$ hyperfine ground state by employing degenerate Raman sideband-cooling ${ }^{30}$. A speciesselective optical lattice ${ }^{31}$ transports the Cs atoms into the Rb cloud. MW radiation prepares the bath atoms in the state $\left|F_{\mathrm{Rb}}=1, m_{F, \mathrm{Rb}}=-1\right\rangle$. The starting point of the heat engine cycle is defined by switching off the optical lattice potential. After a predefined time $t_{i}$, the Cs-Rb interaction is stopped by freezing the positions of the Cs atoms using the optical lattice, and pushing the Rb cloud out of the trap with a resonant laser pulse. State-selective fluorescence imaging of the Cs atoms completes the procedure ${ }^{32}$.

The high-energy and low-energy baths are interchanged by transferring the $\mathrm{Rb}$ atoms from $\left|F_{\mathrm{Rb}}=1, m_{F, \mathrm{Rb}}=-1\right\rangle$ to $\left|F_{\mathrm{Rb}}=1, m_{F, \mathrm{Rb}}=+1\right\rangle$ and vice versa using two successive Landau-Zener sweeps. The transfer takes $\sim 4.4 \mathrm{~ms}$, which is fast 
$N_{\mathrm{SE}}$

1223045678091011

12



b

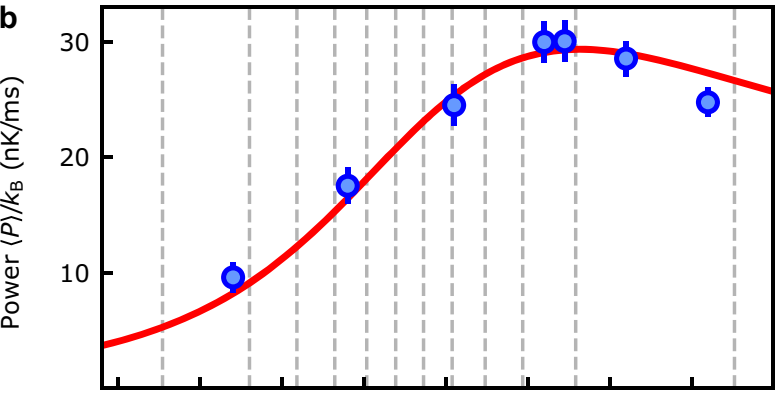

C

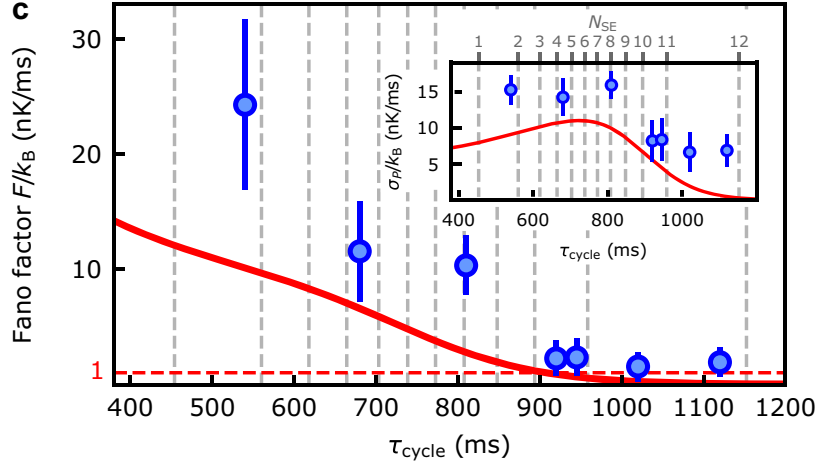

Fig. 3 Performance of the quantum heat engine. a Efficiency $\eta$, Eq. (2) (blue dots), and internal (dissipationless) efficiency $\eta_{\text {int }}$ (green diamonds) for different cycle times; dashed lines indicate the respective expected values. b Average power output, Eq. (3) (blue dots: experimental data, red solid line: theoretical model), with maximal value reached after almost 12 spin exchange collisions. c Fano factor, Eq. (4), and time-resolved fluctuations $\sigma_{P}$ (inset). In all cases, the dashed vertical lines (upper axis) indicate the number of spin-exchange collisions $N_{\mathrm{SE}}$. The different durations between two successive spin-exchange collisions originate from different atomic transition rates ${ }^{14}$. Error bars show statistical uncertainty of $\pm 1 \sigma$ standard deviation.

enough to avoid spin-exchange interactions during the state change of the bath. The two magnetic fields $B_{1}$ and $B_{2}$ defining the quantization axis for the engine operation, are measured using $\mathrm{Rb}$ microwave spectroscopy on the $\mid F_{\mathrm{Rb}}=$ $\left.1, m_{F, \mathrm{Rb}}=0\right\rangle \rightarrow\left|F_{\mathrm{Rb}}=2, m_{F, \mathrm{Rb}}=+1\right\rangle$ transition. The population of the $\mathrm{Rb}$ atoms in state $\left|F_{\mathrm{Rb}}=2, m_{F \mathrm{Rb}}=+1\right\rangle$ is detected by standard absorption imaging, using a time-of-flight measurement (Fig. 4). We fit the measured data with a standard model to extract the transition frequency, which translates into a magnetic field value using the Breit-Rabi formula ${ }^{33}$. We find typical errors of the order of $0.1 \mathrm{mG}$

The magnetic field changes extracting work of the engine have to be adiabatic, i.e., preserving the populations $p_{n}$. The adiabaticity condition writes $\omega_{\text {lar }} / \omega_{\text {lar }}^{2} \ll 1$, where $\omega_{\text {lar }}=\left|g_{F}^{\mathrm{Rb}}\right| \mu_{\mathrm{B}} B / \hbar$ is the Larmor frequency. It can, therefore, be expressed as

$$
A \equiv \frac{\hbar \dot{B}}{\left|g_{F}\right| \mu_{\mathrm{B}} B^{2}} \ll 1 .
$$

Experimentally, we linearly vary the magnetic field from $B_{1}=346.5 \pm 0.2 \mathrm{mG}$ to $B_{2}=31.6 \pm 0.1 \mathrm{mG}$ in a time scale of $10 \mathrm{~ms}$, yielding values of $A\left(B_{1}\right)=0.2 \times 10^{-3}$ and $A\left(B_{2}\right)=14 \times 10^{-3}$, thus fulfilling the adiabatic condition at any time during the variation of the magnetic field. Moreover, the time scale of the magnetic field variation is faster than the time scale associated with the spin exchange collisions (see number of collisions over time in Fig. 3). Hence, the populations $p_{n}$ are constant during the isentropic processes $(\mathrm{B} \rightarrow \mathrm{C}$ and $\mathrm{D} \rightarrow \mathrm{A})$.



b

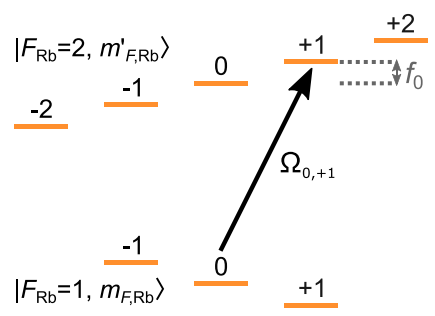

Fig. 4 Magnetic field extraction. a Rb microwave spectra for extraction of the magnetic fields $B_{1}$ and $B_{2}$. Center illustrates the engine cycle and the corresponding Zeeman energy splitting of a Rb bath atom. Red lines correspond to the theory curves and blue dots are experimental data. These measurements yielding magnetic fields $B_{1}=346.5 \pm 0.2 \mathrm{mG}$ and $B_{2}=31.6$ $\pm 0.1 \mathrm{mG}$. Measured spectra confirm similar magnetic fields for $B$ and $C$. b Corresponding microwave transition scheme in the $\mathrm{Rb}$ ground-state hyperfine manifold.

Microscopic model and number of collisions. The quantum heat exchange between engine and bath is based on the understanding of individual spinexchange collisions. In general, the spin-collision rate $\Gamma^{m_{F} \rightarrow m_{F} \pm 1}$ is different both for every initial state $m_{F}$ and for the direction, i.e., $\Delta m_{F}= \pm 1$. The individual rates are well known from coupled-channel calculations of the molecular interaction potential between $\mathrm{Rb}$ and $\mathrm{Cs}^{14}$. These rates allow us to describe the evolution with a rate model ${ }^{21}$ that captures the spin dynamics and yields excellent agreement with the experimental data. From these rates, we also compute the mean number of spin collisions $N_{\text {spin }}$ within a cycle duration $t=t_{\mathrm{D}}$ in two steps. First, we calculate the time-averaged collision rate as the sum of time-averaged collision rates during heating (exothermal spin collisions) and cooling (endothermal spin collisions) as

$$
\begin{aligned}
\langle\Gamma(t)\rangle= & \left\langle\Gamma_{\mathrm{A} \rightarrow \mathrm{B}}(t)\right\rangle+\left\langle\Gamma_{\mathrm{C} \rightarrow \mathrm{D}}(t)\right\rangle \\
= & \sum_{m_{F}=+3}^{-2} p_{m_{F}}(t) \Gamma_{\mathrm{A} \rightarrow \mathrm{B}}^{m_{F} \rightarrow m_{F}-1} \\
& +\sum_{m_{F}=+2}^{-3} p_{m_{F}}(t) \Gamma_{\mathrm{C} \rightarrow \mathrm{D}}^{m_{F} \rightarrow m_{F}+1}
\end{aligned}
$$

Second, we integrate these rates during the heating and cooling to obtain the number of collisions within cycle time $t$ as

$$
\begin{aligned}
N_{\text {spin }}(t) & =N_{\mathrm{A} \rightarrow \mathrm{B}}+N_{\mathrm{C} \rightarrow \mathrm{D}} \\
& =\int_{0}^{t_{\mathrm{B}}}\left(\left\langle\Gamma_{\mathrm{A} \rightarrow \mathrm{B}}\left(t^{\prime}\right)\right\rangle d t^{\prime}+\int_{t_{\mathrm{C}}}^{t_{\mathrm{D}}}\left\langle\Gamma_{\mathrm{C} \rightarrow \mathrm{D}}\left(t^{\prime}\right)\right\rangle\right) d t^{\prime} .
\end{aligned}
$$

In order to close the cycle, the inital and final Cs states before and after a cycle have to be the equal, leading to the condition $N_{\mathrm{A} \rightarrow \mathrm{B}}=N_{\mathrm{C} \rightarrow \mathrm{D}}$.

Efficiency of the endoreversibe machine. We calculate the efficiency by distinguishing two different forms of heat exchange. First, we consider the respective mean energies given $\left(\left\langle Q_{1}\right\rangle\right)$ and taken $\left(\left\langle Q_{2}\right\rangle\right)$ by the baths, where $\left\langle Q_{1}\right\rangle-\left|\left\langle Q_{2}\right\rangle\right|$ is the energy turnover of the reservoirs per cycle. Second, we consider the average energies absorbed $\left(\left\langle Q_{H}\right\rangle\right)$ and rejected $\left(\left\langle Q_{C}\right\rangle\right)$ from the engine, where $\left\langle Q_{H}\right\rangle-\left|\left\langle Q_{C}\right\rangle\right|$ is the energy turnover of the machine. Both quantities differ because of the different atomic Landé factors of $\mathrm{Cs}$ and $\mathrm{Rb}$. The difference $\left\langle Q_{\mathrm{L}}\right\rangle=\left(\left\langle Q_{1}\right\rangle-\left|\left\langle Q_{2}\right\rangle\right|\right)-$

$\left(\left\langle Q_{\mathrm{H}}\right\rangle-\left|\left\langle Q_{\mathrm{C}}\right\rangle\right|\right)$ is dissipated via elastic collisions and irreversibly lost to the kinetic energy of $\mathrm{Rb}$. We macroscopically model it as a heat leak from the high-energy reservoir. Using the population distribution of the quasi-spin levels at the cycle 
points in Fig. 2 of the main text, the individual heats can be calculated, leading to

$$
\begin{aligned}
\left\langle Q_{\mathrm{L}}\right\rangle= & \left(\left\langle Q_{1}\right\rangle-\left|\left\langle Q_{2}\right\rangle\right|\right)-\left(\left\langle Q_{\mathrm{H}}\right\rangle-\left|\left\langle Q_{\mathrm{C}}\right\rangle\right|\right) \\
= & \left(\sum_{n} n\left[p_{n}^{\mathrm{B}}-p_{n}^{\mathrm{A}}\right] \kappa B_{1}-\left|\sum_{n} n\left[p_{n}^{\mathrm{D}}-p_{n}^{\mathrm{C}}\right] \kappa B_{2}\right|\right) \\
& -\left(\sum_{n} n\left[p_{n}^{\mathrm{B}}-p_{n}^{\mathrm{A}}\right] \lambda B_{1}-\left|\sum_{n} n\left[p_{n}^{\mathrm{D}}-p_{n}^{\mathrm{C}}\right] \lambda B_{2}\right|\right) .
\end{aligned}
$$

Owing to preservation of populations during adiabatic strokes, we can further use $p_{n}^{\mathrm{D}}=p_{n}^{\mathrm{A}}$ and $p_{n}^{\mathrm{B}}=p_{n}^{\mathrm{C}}$, yielding the expression for the dissipated heat

$$
\left\langle Q_{\mathrm{L}}\right\rangle=\sum_{n} n\left(p_{n}^{B}-p_{n}^{A}\right)(\kappa-\lambda)\left(B_{1}-B_{2}\right) .
$$

The efficiency is calculated as the work, $|\langle W\rangle|=\left\langle Q_{\mathrm{H}}\right\rangle-\left|\left\langle Q_{\mathrm{C}}\right\rangle\right|$, produced by the engine, divided by the energy provided by the high-energy bath, $\left\langle Q_{\mathrm{H}}\right\rangle+\left\langle Q_{\mathrm{L}}\right\rangle$. Using $p_{n}^{\mathrm{D}}=p_{n}^{\mathrm{A}}, p_{n}^{\mathrm{B}}=p_{n}^{\mathrm{C}}$ and $\gamma=\lambda / \kappa$, we find

$$
\eta=\frac{\left\langle Q_{\mathrm{H}}\right\rangle-\left|\left\langle Q_{\mathrm{C}}\right\rangle\right|}{\left\langle Q_{\mathrm{H}}\right\rangle+\left\langle Q_{\mathrm{L}}\right\rangle}=\frac{\gamma\left(B_{1}-B_{2}\right)}{B_{1}-B_{2}+\gamma B_{2}} .
$$

The internal efficiency of the engine is computed as the ratio of the produced work $|\langle W\rangle|$ and the heat absorbed by the machine $\left\langle Q_{\mathrm{H}}\right\rangle$ :

$$
\eta_{\text {int }}=\frac{\left\langle Q_{\mathrm{H}}\right\rangle-\left|\left\langle Q_{\mathrm{C}}\right\rangle\right|}{\left\langle Q_{\mathrm{H}}\right\rangle}=1-\frac{B_{2}}{B_{1}} .
$$

It corresponds to the efficiency without a leak $(\gamma=1)$.

Fluctuations of the quantum machine. To extract the fluctuations of the engine, Eq. (4), we calculate the mean power, Eq. (3), via $\langle P\rangle=|\langle W\rangle| \tau_{\text {cycle. }}$ The cycle time $\tau_{\text {cycle }}=t_{\mathrm{D}}$ is experimentally controlled, and we assume that it is a fixed parameter not adding further fluctuations to the power-output fluctuations. Therefore, we can restrict the calculation to the fluctuations $\sigma_{W}$ of work $\langle W\rangle$ as $\sigma_{W}^{2}=\left\langle W^{2}\right\rangle-\langle W\rangle^{2}$. The work is given by the difference of energy absorbed by and rejected from the engine $|\langle W\rangle|=\left\langle Q_{H}\right\rangle-\left|\left\langle Q_{\mathrm{C}}\right\rangle\right|$, and hence

$$
\begin{aligned}
\sigma_{W}^{2} & =\sigma_{Q_{\mathrm{H}}}^{2}+\sigma_{Q_{\mathrm{C}}}^{2} \\
& =\left\langle Q_{\mathrm{H}}^{2}\right\rangle-\left\langle Q_{\mathrm{H}}\right\rangle^{2}+\left\langle Q_{\mathrm{C}}^{2}\right\rangle-\left\langle Q_{\mathrm{C}}\right\rangle^{2} .
\end{aligned}
$$

The averages and variances of heat absorbed or rejected depend on the energy differences at the different points during the cycle, for example, $\left\langle Q_{\mathrm{H}}\right\rangle=E\left(t_{\mathrm{B}}, B_{1}\right)-$ $E_{0}\left(t_{0}, B_{1}\right)$. Here, $E\left(t_{i}, B_{j}\right)=\sum_{n} p_{n}^{i}\left(t_{i}\right) n \lambda B_{j}$ can be computed from the measured populations $\left\{p_{n}^{i}\right\}$ of level $n$ at point $i=\mathrm{A}, \mathrm{B}, \mathrm{C}, \mathrm{D}$ during the cycle and the magnetic field $B_{j}(j=1,2)$, together with mean energy and variance. Then, the fluctuations $\sigma_{Q}^{2}$ of heat $\langle Q\rangle$ exchanged when changing the engine's probability distribution from point $i$ to point $f$ at a magnetic field $B_{j}$ reads

$$
\begin{aligned}
\sigma_{Q}^{2}= & \sum_{n}\left(p_{n}^{f}\left(t_{f}\right)+p_{n}^{i}\left(t_{i}\right)\right)\left(n \lambda B_{j}\right)^{2} \\
& -\left\{\left[\sum_{n} p_{n}^{f}\left(t_{f}\right) n \lambda B_{j}\right]^{2}+\left[\sum_{n} p_{n}^{i}\left(t_{i}\right) n \lambda B_{j}\right]^{2}\right\},
\end{aligned}
$$

where, using the notation of Fig. $1 \mathrm{~b}$, for $\left\langle Q_{\mathrm{H}}\right\rangle i=0, f=\mathrm{B}$, and $B_{j}=B_{1}$, and for $\left\langle Q_{\mathrm{C}}\right\rangle$ $i=\mathrm{C}, f=\mathrm{D}$, and $B_{j}=B_{2}$. Inserting these expressions into Eq. (12) allows us to compute the work fluctuations for every cycle time $\tau_{\text {cycle }}=t_{\mathrm{A}}$ and thereby the variance of the output power fluctuations $\sigma_{p}^{2}$.

\section{Data availability}

The data that support the plots and findings of this study are available from the corresponding author upon reasonable request.

Received: 18 November 2020; Accepted: 5 March 2021;

Published online: 06 April 2021

\section{References}

1. Cengel, Y. A. \& Boles, M. A. Thermodynamics. An Engineering Approach (McGraw-Hill, 2001)

2. Andresen, B., Salamon, P. \& Berry, R. S. Thermodynamics in finite time. Phys. Today. 37, 62 (1984).

3. Andresen, B. Current trends in finite-time thermodynamics. Angew. Chem. Int. Ed. 50, 2690 (2011).

4. Pietzonka, P. \& Seifert, U. Universal trade-off between power, efficiency and constancy in steady-state heat engines. Phys. Rev. Lett. 120, 190602 (2018).

5. Holubec, V. \& Ryabov, A. Cycling tames power fluctuations near optimum efficiency. Phys. Rev. Lett. 121, 120601 (2018).

6. Denzler, T. \& Lutz, E. Power fluctuations in a finite-time quantum Carnot engine. Preprint at https://arxiv.org/abs/2007.01034 (2020).

7. Rossnagel, J. et al. A single-atom heat engine. Science 352, 325-329 (2016).
8. von Lindenfels, D. et al. Spin heat engine coupled to a harmonic-oscillator flywheel. Phys. Rev. Lett. 123, 080602 (2019).

9. Van Horne, N. et al. Single-atom energy-conversion device with a quantum load. NPJ Quantum Information 6, 37 (2020).

10. Klatzow, J. et al. Experimental demonstration of quantum effects in the operation of microscopic heat engines. Phys. Rev. Lett. 122, 110601 (2019).

11. de Assis, R. J. et al. Efficiency of a quantum otto heat engine operating under a reservoir at effective negative temperatures. Phys. Rev. Lett. 122, 240602 (2019).

12. Peterson, J. P. S. et al. Experimental characterization of a spin quantum heat engine. Phys. Rev. Lett. 123, 240601 (2019).

13. Kosloff, R. \& Rezek, Y. The quantum harmonic otto cycle. Entropy 19, 136 (2017).

14. Schmidt, F. et al. Tailored single-atom collisions at ultra-low energies. Phys. Rev. Lett. 122, 013401 (2019)

15. Sikorsky, T., Meir, Z., Ben-shlomi, R., Akerman, N. \& Ozeri, R. Spincontrolled atom-ion chemistry. Nat. Commun. 9, 920 (2018).

16. Kosloff, R. \& Feldmann, T. Discrete four-stroke quantum heat engine exploring the origin of friction. Phys. Rev. E 65, 055102(R) (2002).

17. Plastina, F. et al. Irreversible work and inner friction in quantum thermodynamic processes. Phys. Rev. Lett. 113, 260601 (2014).

18. Esposito, M., Harbola, U. \& Mukamel, S. Nonequilibrium fluctuations, fluctuation theorems, and counting statistics in quantum systems. Rev. Mod. Phys. 81, 1665-1702 (2009).

19. Friedman, H. M., Agarwalla, B. K. \& Segal, D. Quantum energy exchange and refrigeration: a full-counting statistics approach. New J. Phys. 20, 083026 (2018).

20. Cohen-Tannoudji, C. \& Guery-Odelin, D. Advances in Atomic Physics (World Scientific, Singapore, 2011).

21. Bouton, Q. et al. Single-atom quantum probes for ultracold gases boosted by nonequilibrium spin dynamics. Phys. Rev. X 10, 011018 (2020).

22. Masser, R. \& Hoffmann, K. H. Dissipative endoreversible engine with given efficiency. Entropy 21, 1117 (2019).

23. Fano, U. Ionization yield of radiations. II. The fluctuations of the number of ions. Phys. Rev. 72, 26-29 (1947).

24. Abah, O. \& Lutz, E. Efficiency of heat engines coupled to nonequilibrium reservoirs. EPL 106, 20001 (2014)

25. Alicki, R. \& Gelbwaser-Klimovsky, D. Non-equilibrium quantum heat machines. New J. Phys. 17, 115012 (2015).

26. Rossnagel, J., Abah, O., Schmidt-Kaler, F., Singer, K. \& Lutz, E. Nanoscale heat engine beyond the Carnot limit. Phys. Rev. Lett. 112, 03602 (2014).

27. Klaers, J., Faelt, S., Imamoglu, A. \& Togan, E. Squeezed thermal reservoirs as a resource for a nanomechanical engine beyond the Carnot limit. Phys. Rev. X 7 , 031044 (2017).

28. Thomas, G., Siddharth, N., Banerjee, S. \& Ghosh, S. Thermodynamics of nonMarkovian reservoirs and heat engines. Phys. Rev. E 97, 062108 (2018),

29. Pezzutto, M., Paternostro, M. \& Omar, Y. An out-of-equilibrium nonMarkovian quantum heat engine. Quantum Sci. Technol. 4, 025002 (2019).

30. Kerman, A. J., Vuletić, V., Chin, C. \& Chu, S. Beyond optical molasses: 3D raman sideband cooling of atomic cesium to high phase-space density. Phys. Rev. Lett. 84, 439 (2000).

31. Schmidt, F. et al. Precision measurement of the ${ }^{87} \mathrm{Rb}$ tune-out wavelength in the hyperfine ground state $F=1$ at $790 \mathrm{~nm}$. Phys. Rev. A 93, 022507 (2016).

32. Schmidt, S. et al. Quantum spin dynamics of individual neutral impurities coupled to a Bose-Einstein condensate. Phys. Rev. Lett. 121, 1304031 (2018).

33. Haken, H. \& Wolf, H. C. The Physics of Atoms and Quanta, Introduction to Experiments and Theory (Springer, 2005).

\section{Acknowledgements}

We thank E. Tiemann for providing us with the scattering cross-sections underlying our numerical model, and T. Busch and J. Anglin for helpful comments on the manuscript. This work was funded by Deutsche Forschungsgemeinschaft via Sonderforschungsbereich (SFB) SFB/TRR185 (Project No. 277625399) and Forschergruppe FOR 2724.

\section{Author contributions}

Q.B. and J.N. contributed equally to this work. A.W. and Q.B. conceived the experiment and supervised the project. Q.B., J.N., S.B., and D.A. performed the experimental measurements. J.N., S.B., and Q.B. contributed to the microscopic numerical model. E.L. provided the theoretical thermodynamic explanation. All authors contributed to analysis and interpretation of the data, and writing of the manuscript.

\section{Funding}

Open Access funding enabled and organized by Projekt DEAL. 


\section{Competing interests}

The authors declare no competing interests.

\section{Additional information}

Correspondence and requests for materials should be addressed to A.W.

Peer review information Nature Communications thanks the anonymous reviewers for their contribution to the peer review of this work.

Reprints and permission information is available at http://www.nature.com/reprints

Publisher's note Springer Nature remains neutral with regard to jurisdictional claims in published maps and institutional affiliations. (c) (i) Open Access This article is licensed under a Creative Commons Attribution 4.0 International License, which permits use, sharing, adaptation, distribution and reproduction in any medium or format, as long as you give appropriate credit to the original author(s) and the source, provide a link to the Creative Commons license, and indicate if changes were made. The images or other third party material in this article are included in the article's Creative Commons license, unless indicated otherwise in a credit line to the material. If material is not included in the article's Creative Commons license and your intended use is not permitted by statutory regulation or exceeds the permitted use, you will need to obtain permission directly from the copyright holder. To view a copy of this license, visit http://creativecommons.org/ licenses/by/4.0/

(C) The Author(s) 2021 